\title{
Survival and Hazard Model Analysis of Breastfeeding Variables on Return to Postpartum Amenorrhea in Rural Mada Women of Central Nigeria.
}

\author{
Dansadiq Adam Ishaya ${ }^{1}$, Husseini Garba Dikko ${ }^{2}$ \\ ${ }^{1}$ (Department of Mathematics and Statistics, Nasarawa State Polytechnic, Lafia. Nigeria.) \\ 2 (Department of Mathematics, Faculty of Science, Ahmadu Bello University, Zaria. Nigeria.)
}

\begin{abstract}
An examination of breastfeeding and postpartum amenorrhea survey was carried out on 196 rural Mada women in central Nigeria. It was realized that, age, educational background and religious belief does not affect the duration of postpartum amenorrhea (PPA). The survival function of subjects decreases as the duration of breastfeeding without resuming menses increases, while, the hazard function increases as the duration of breastfeeding without return menses increases. Also, the duration of PPA varies from one woman to another. The study also shows that the association between breastfeeding and PPA does not necessarily indicate that duration of breastfeeding affect the duration of PPA, but could be that the two are control by some physiological effect of hormones.
\end{abstract}

Keywords: Amenorrhea, Breastfeeding, Fertility, Hazard, Postpartum, Survival.

\section{Introduction}

Demographic researches in many part of the world continue to yield a result which consistently demonstrates that breastfeeding is associated with postpartum amenorrhea (PPA), reduce fecund ability and increase long birth interval. Some earlier studies, [1] provides empirical evidence that showed that, at least at the aggregate level, when regressing average durations of PPA on average durations of breastfeeding, the two are significantly and positively related.

Postpartum amenorrhea is the time elapsed between the termination of pregnancy and the resumption of menses just after delivery. It is the most important factor for female's reproductive life. The duration of PPA has been found to also be positively related with the nutritional status of women,. It is also well established that the relationship between breastfeeding and postpartum amenorrhea depends heavily on the effectiveness of the nursing stimulus. In fact, when breastfeeding is prolonged and intensive, the average PPA may last from one to two years, [2].

Breastfeeding is the act of feeding a child breast milk directly from the breast to mouth. In early Egyptian, Greek and Roman Empire women usually feed only their own children. However, breastfeeding began to be seen as something too common to be done by royalty and wet nurses were employed to breastfeed the children of the royal families. These were extended over the ages particularly in the west Europe. This practice was later discouraged and every woman was encouraged to breastfeed her child. Japan became the first developed country to have a Baby friendly hospital in 2006. The Baby Friendly Hospital Initiative (BFHI) is a World Health Organization and UNICEF launched in 1991, following the adoption of the Innocent declaration on breastfeed promotion in 1990. The initiative is a global effort for improving the role of maternity service to enable mothers to breastfeed babies for the best start in life. It aims at improving the case of pregnant women, mothers and new born at health facilities that provides maternity services for protecting, promoting and supporting breastfeeding in accordance with the international code of marketing of breast milk substitute. UNICEF, WHO and many national government health agencies recommended that babies should be breastfed exclusively for their first 6 months of life. The BHFI aims to increase the numbers of babies who are exclusively breastfed worldwide; a goal which the WHO estimates could contribute to avoiding over a million child death in a year, [3].

The initiative is not being practice by most of the Mada women because; A malnourished woman does not have the strength to breastfeed a baby exclusively for a long time since she needs good diet for all body system to function effectively. As such, early supplemented type of breastfeeding is the major type of breastfeeding among Mada women. The women are engaged in hard labour in the field with their babies exposed to heat and dehydrated. Because of these reasons, most Mada women tend to supplement breastfeeding with water and other food to reduce the rate of suckling' sucking and to replace the dehydrated water during the day work.

Researchers have shown that breastfeed babies are less likely to suffer from serious illnesses such as gastroenteritis, asthma and even respiratory infections. Adults who were breastfed as babies are less likely to 
develop risk factor for heart disease such as obesity and high blood pressure. There are benefits for the mothers too: women who breastfeed can promote hormonal processes that protect mothers against postpartum amenorrhea depression, by helping the regulation of sleep and wake pattern for mother and child, improving mother's self-efficacy and her emotional involvement with the child, reducing the child temperamental difficulties and promoting a better interaction between mother and child. They further claimed that, frequent and intensive breastfeeding lower risk of developing breast cancer and ovarian cancer among many other diseases later in life. The exclusive breastfeeding method of birth control is called the Lactation Amenorrhea Method of birth control (LAM). (Lactation amenorrhea refers to the natural postpartum infertility that occurs when a woman is not menstruating). Exclusive breastfeeding has in fact shown to be an excellent form of birth control, but only when certain criteria are met (the breastfeeding must be frequently, intensively and within the first six months of initiation), [4].

It is known that African women have the highest fertility rate in the world. This has been made feasible through the increased availability of demography survey data. There are several factors responsible for this high level of fertility. Some of these include early marriage, infant mortality, lack of experience in the use of modern contraceptive, high values associate with numbers of children an individual has. The fear that some of the children may die early in life make many Africans to give birth to plenty children. In polygamy families there is competition among the co-wives which eventually result in high fertility in Africa, [5].

\subsection{Literature}

\section{Review of Literature}

Many researchers in the area of breastfeeding and postpartum amenorrhea have produced consistent results which agree with one another that breastfeeding is associated with return menses and ovulation, reduce fecundity and prolong birth intervals. Some works of earlier researchers in demography provides empirical evidence accumulated through the years, which showed that, at least at the aggregate level, when regressing average duration of postpartum amenorrhea on average duration of breastfeeding, the two are significantly and positively related, [6].

Researches in this area have been strongly influenced by the work of Jean - Pierre Habicht and colleagues, (we shall refer to this as Habicht model in the rest of this study). Their article was one of the first to question the essentially correlation approach of previous investigations. They pointed out that it is incorrect to use the total duration of breastfeeding as an explanatory variable when, in most developing countries settings, amenorrhea typically ends well before breastfeeding is terminated, The Habicht model represents an important milestone in demography research for another reason as well, in that it was one of the first attempts to integrate information on breastfeeding behavior with the underlying physiology of human lactation in other to gain a greater understanding of the demography consequences of lactation and breastfeeding. Although the model today is seen to have some limitations (for example, it assumes a threshold level for key hormones above which nursing mothers are not fecund, and it assumed incorrectly that this hormone is prolactin), it nonetheless retains many important factors of the return of postpartum fecundity as it is understood today. One good thing about Habicht model is that, the model assumes that the time to resumption of menses once breastfeeding has been discontinued is the same no matter when during the postpartum period breastfeeding is stopped. The statistical analysis went ahead to provide an estimate of 1 to 2 months for the time from the discontinuation of breastfeeding to first occurrence of menses, [7]. Thus, simply knowing that the duration of breastfeeding is highly correlated with the duration of postpartum amenorrhea reveals little about the underlying causal relationship between the two. This is the case whether or not; aggregate or individual level data is used in regression analysis.

The experience of breastfeeding is special for so many reasons; joyful bonding between mother and child, the cost saving and the health benefits for both mother and baby. One major important of breastfeeding is that it protects babies. Early breast milk commonly refers to as liquid gold, colostrums is the tick yellow first breast milk that is made during pregnancy and just after birth. This milk is very rich in nutrients and antibodies to protect an infant baby. In their world survey of the timing of breastfeeding claimed that, because of the cultural believes in some societies that first breast milk is unclean; there is usually a delay breastfeeding for about 48 hours before initiation of breastfeeding, [8]. The importance of breastfeeding in regulating individual and social fertility has been a matter of general interest for many years because it tends to increase the average birth interval and therefore to reduce women's fertility over low life span especially in societies where the use of contraceptive methods are not common, When a baby sucks on the nipple, the stimulation is transmitted to the pituitary gland and hormones called prolactin conveys commands to change blood into breast milk and oxytocine operate to push out the milk that accumulates in the breast ducts. The health condition of a breastfeeding mother, malnutrition and psychological problems are responsible for poor breastfeeding in woman, [9]. 


\subsection{Determinant of Postpartum Amenorrhea}

There are four mechanism related to PPA in breastfeeding women. Two of them "Weaning and Infant Mortality" have a direct causal effect. They terminate nursing episode and therefore the hormonal stimulus that child suckling has on the delay of fecund menses cycle. The third mechanism is related to the breastfeeding patterns prevalent among those mothers who menstruated while they were still breastfeeding. The fourth causal factor, maternal nutrition and health status, may also modify the hormonal stimulus by delaying the resumption of normal menstrual cycles, [10].

The Habicht model, just like other researches in the field proposed on physiological mechanism for postpartum amenorrhea involving the pituitary hormone prolactin (PRL), in the model, the authors assumed that reoccurrence of PPA occur when the plasma level of the main hormone PRL fall below a threshold value. However, the mechanism of PPA lactation infertility does not involve PRL directly, such as beta-endorphin contrary to earlier studies which proposed that it was responsible for the amenorrhea of nursing mothers. Instead it is the direct suckling induced failure of the central mechanisms to secret required hormones in normal frequencies that is directly responsible for postpartum lactation infertility, [11].

According to, [12]. Other mechanisms like the physiological mechanisms underlying these observed relationship originate in the hypothalamus, which is located in the brain. The woman's hypothalamic nuclei release gonadotropin -releasing hormones $(\mathrm{GnRH})$ in a regular, episodic fashion, which in turn trigger the release of pulsatile and episodic release of luteinizing hormones (LH) and follicle stimulating hormone (FSH) from the pituitary gland. This biological process is known as the hypothalamic pulse generator (HPG). The release of certain amounts of luteinizing hormones has been found to be crucial for normal ovarian activity and fecund menstrual circle. Recent study has also shown that normal pattern of GnRH and LH release can be disrupted by external factors, such as heavy physical exercise or activity, or physiological stress, in addition to breastfeeding. In the case of the breastfeeding women during the postpartum period, however, the suckling stimulus may be the primary signal which disrupts the normal pattern of hormonal activity. But the disruption of the HPG neuron is a common characteristic of altered GnRH and LH release, and thus of amenorrhea and fertility. This general mechanism represents a direct link between the external environment and the internal regulation of the reproductive system,

Some researchers made important attempt to investigate the effect of women breastfeeding and nutritional states on PPA. Just a handful of the studies have investigated the corresponding effects of breastfeeding patterns such as; frequency of breastfeeding and intensity alone side nutrition on PPA. When the potential confounding factor (like age, parity, work pattern, health status, education are under control), [13].

Biological and Demography literature all confirmed one another that breastfeeding plays a great role in increasing the duration of PPA and the common conclusion always drawn is that, Lactation increases the duration of PPA and that long-term breastfeeding is statistically associated with long-term PPA ovarian inactivity and reduce fecundity. However, It is incorrect to use the total duration of breastfeeding as an explanatory variable when, in most developing countries settings, amenorrhea typically ends well before breastfeeding is terminated. Thus, simply knowing that the duration of breastfeeding is highly correlated with the duration of PPA reveals little about the underlying causal relationship between the two. This is the case whether or not aggregate or individual level data is used in the regression analysis, [7].

Expressing doubt about the usefulness of correlation approaches, [14]. She pointed out the unsound argument in the assumptions that the relationship between breastfeeding and PPA is unidirectional, that is, that breastfeeding affect amenorrhea does not mean amenorrhea affect breastfeeding. She also highlighted the cases where some women may stop nursing soon after their menses returns, while others may stop breastfeeding soon after they recognize they are pregnant following a previous birth. In these situations, the duration of breastfeeding is a function of the duration of PPA and not the reverse.

Confirmed that all other factors being equal, [15], the association between the duration of Postpartum Amenorrhea and breastfeeding is not due to the direct effect of prolonged lactation implies by the strong correlation between the two variables. It is clearly that the duration of postpartum amenorrhea is less than the duration of breastfeeding over the first 24 months following birth. That is lactation outlast amenorrhea. Second, the time of resumption of ovulation and or menses once suckling has been discontinued before menses resume is the same no matter when and for what (either infant death or weaning) during postpartum period nursing is discontinued. Third, the time it takes to experience the first postpartum menses following the discontinuation of breastfeeding is approximately the same time it takes normal reproductive function to return following birth when the mother for whatever reasons chooses not to breastfeed at all. Fourth, the association between breastfeeding and duration of PPA is determined by the combined additive effects of nursing intensities (which they defined as average nursing frequency per $24 \mathrm{hrs}$ ), the timing of the introduction of supplement, the timing of weaning and infant death if these events occur, and possible other. 


\subsection{Method}

\section{Methodology}

Nasarawa state is a state in central region of Nigeria which is characterized by multi-ethnic and varying cultural background that varies from one ethnic group to another. Akwanga is a Local government in Nasarawa state Nigeria and is the homes of Mada people, with Kokona in the west of Akwanga, Sanga local government of Kaduna state boundary the local governments in the North, while Wamba boundary Akwanga in the east and Nasarawa-Eggon on the south of Akwanga. All tribes in the local government surrounding Akwanga and Kokona have similar life style just like the Mada people. The Mada people are predominantly farmers, not many of them are educated but most are moderately exposed to modern socialization. The economic factors contribute highly to the life style of people of Mada just as in the case of most developing countries. Their women are seen to be as productive as the men, as such; the rural Mada women are engaged in hard labour such as farming. Since farming is the only source of income to most Mada people, the welfare and education of their children all repose on the output from their farms.

Some villages across Akwanga and Kokona would be selected as target areas where sample for this study would be obtained. The village heads and husbands of every woman selected for the research would be notified of the survey and its purpose. The villages to be sampled are: Gwanje, Andaha, Moroa, Ungwan-Zaria, Ningo and Tatara. The respondents are the women who have given birth and wean at least once or have experience infant death during breastfeeding. Women who are breastfeeding their first children are not considered. A sample of 200 women will be selected for this survey from the population of all the women that have met the requirements: A woman is expected to have breastfed and wean at least a child or have experience infant mortality which led to resume menses. The sampling techniques used for this study is the simple random sampling techniques.

From the termination of pregnancy, the period before the resumption of menses should be consider as a waiting time, and the occurrence of menses consider as an event. These ideas will be used to study the survival function and the hazard function for postpartum amenorrhea. Looking at the process of moving from the state of not seeing menses (PPA) to another state of seeing menses (resumption of menses), it clearly shows that the process follows a stochastic process, thus the researcher intend to derive a survival and hazard model. The data obtained from this research work would be used to check for the validity of the models that will be derived.

\subsection{Modeling Strategy}

The main objectives of this research work are; to develop a model to be used in estimating time-toevent for a group of individuals. To estimate time-to-event for a group of individuals (that is, time until postpartum amenorrhea is experience for a group of mothers) and the average duration of postpartum amenorrhea after weaning and or infant mortality.

Let $\mathrm{T}$ be a non-negative random variable representing the waiting time until the occurrence of an event (resumption of menses). For simplicity, we will adopt the terminology of survival analysis, referring to event of interest as "resumption of menses" and the waiting time as "survival" time. We will assume for now that $\mathrm{T}$ is a continuous random variable with probability density function (p.d.f.) $f(t)$ and cumulative distribution function (c.d.f.) $\mathrm{F}(\mathrm{t})=\operatorname{Pr}\left(\mathrm{T}_{\mathrm{t}}\right)$, giving the probability that the event has occurred (resumption of menses) at time $t$. For convenience purpose, we will work with the complement of the c.d.f, which is the survival function.

$$
S(t)=\operatorname{Pr}(T>t)=1-F(t)=f(x) d x
$$

This is the probability of surviving (being in PPA) at period $t$, or in simple form, the probability that the event of interest (resumption of menses) has not occurred. This is known as the survival function.

The hazard function is an alternative characteristic of the distribution of $\mathrm{T}$, or instantaneous rate of occurrence of the event (resumption of menses), and is defined as,

$$
h(t)=\lim _{d t \rightarrow 0} \frac{p(t \leq T \leq t+d t / T \geq t)}{d t}
$$

The numerator of this expression is the conditional probability that the event will occurr in the interval $(t, t+d t)$ given that it has not occurred before, and the denominator is the width of the interval. When the numerator is divided by the denominator, we obtained a rate of event occurring per unit time (rate of resumption of menses per month). Taking the limit as the width approaches zero, we obtain an instantaneous rate of occurrence. In other words, the hazard function is the probability that if a woman is in PPA period (survive) to time $\mathrm{t}$; she will resume menses in the next instant.

The conditional probability;

$$
p(t \leq T \leq t+d t / T \geq t)
$$


may be written as the ratio of the joint probability that $T$ is in the interval $(t, t+d t)$ and $T>t$ to the probability of the condition $T>t$. The former may be written as $f(t) d t$ for small $d t$, while the latter is $S(t)$ by definition. Hence dividing by $d t$ and obtaining the limit will yield;

$$
h(t)=\frac{f(t)}{S(t)^{\prime}}
$$

which may be consider a hazard function, and it is the rate of resumption of menses at period t equal the density of the occurrence of menses at $t$, divide by the probability of surviving to that period $t$ without resumption of menses.

Our interest here is focus on exponential hazard, hence;

$$
\text { Exponential function } P(T=t)=f(t)=h e^{-h t}
$$

and

$$
\text { Survival function: } \begin{aligned}
P(T & >t)=S(t)=\int_{t}^{\infty} h e^{-h u} d u=-e^{-h u} \\
& =0-\left(-e^{-h t}\right)=\left.e^{-h t}\right|_{t} ^{\infty}
\end{aligned}
$$

The reproductive history of a woman can be treated as a collection of random point over time (i.e. a stochastic process) which is characterized by a sequence of events such as births, infant deaths, initiation and termination of breastfeeding, initiation and termination of PPA, and so on. Collectively, the history is made up of finite number of states, say $k$. Let us assume that a woman stays at every state $j$ a random length of time $\left(\mathrm{T}_{1}\right.$, $\left.\mathrm{T}_{2, \ldots}, \mathrm{T}_{\mathrm{K}}\right)$. And suppose that these random variables are completely characterized by the probability distribution $f\left(t_{j} / x\right)$, where $t_{j}$ is a realization of $\mathrm{T}_{\mathrm{j}}$. One of the basic concepts is that of the "failure" rate (resume menses) or hazard function, $h\left(t_{j}\right)$. The hazard function as earlier defined is the instantaneous probability of resumption of menses at time $t_{j}$ given that the menses has not resume before $t_{j}$. We can now conclude that the hazard function is the rate at which events are completed after period $t_{j}$ given that the events lasted up to period $t_{j},[16]$.

\subsection{Censoring}

Censoring is a form of missing data problem which is common in survival analysis. Ideally, both the beginning and the end date of PPA of a subject (breastfeeding mother) are supposed to be known, in which case the duration of PPA is known. If it is known only that the resumption of menses is after some dates, we refer to this as right censoring. Right censoring will occur for those subjects whose date of beginning of PPA is known but who are still in postpartum period when they are lost to follow-up or when the study ends. If the subject's postpartum period is known to be less than certain duration, then the postpartum period is said to be left censored. It may also happen that subjects with a postpartum period less than a certain threshold may not be observed at all: this is called truncation. Truncation should not be confused with left censoring since for a left censored datum, it is known that the subject exist, but for a truncated datum, we may be completely unaware of the subject, [17].

Another approach to survival analysis is the non-parametric method known as the Kaplan-Meier estimate .The Kaplan-Meier estimate is a non-parametric estimate of survival function with no mathematical assumptions. It is simply the empirical probability of surviving past certain times in the sample (that is, describing survivorship of study population taking into account censoring). Kaplan-Meier estimate is mainly descriptive, does not control for covariates, requires categorical predictors and cannot accommodate time dependent variable, [18].

\section{Results and Discussion}

In developing countries, it is obvious that long duration of breastfeeding is the common practice in most rural areas. This is also true for Mada mothers who participated in this study. $86 \%$ of the women that entered into this study claimed to have breastfed their babies for more than 12 while only $14 \%$ breastfeed their babies for less than 12 months. In general, the average frequency of breastfeeding observed in this study is about 10-15 times per day and the average intensity of the breastfeeding is about 3-6 minutes per nursing episode. $47 \%$ of the population had their menses resumed before terminating breastfeeding while the remaining $53 \%$ terminate breastfeeding before menses resumed. Most of the mothers in this group made use of family planning. Those in the weaning group, have the same duration of resume menses after weaning of about 1-2 months with those in the infant mortality group. Table 1.1 gives the summary of the responses provided by the 196 respondents. 
Table 1.1 Summaries of Responses From Respondents.

\begin{tabular}{|c|c|c|c|}
\hline Items & Response mode & No. of respondents & $\%$ of respondents \\
\hline \multirow[t]{2}{*}{ Resumption of menses } & Before weaning & 92 & $47 \%$ \\
\hline & After weaning & 104 & $53 \%$ \\
\hline \multirow[t]{2}{*}{ Used family planning } & Yes & 66 & $34 \%$ \\
\hline & No & 130 & $66 \%$ \\
\hline \multirow{2}{*}{$\begin{array}{ll}\text { Duration } & \text { of } \\
\text { breastfeeding } & \end{array}$} & 1year or less & 27 & $14 \%$ \\
\hline & More than 1year & 169 & $86 \%$ \\
\hline \multirow{2}{*}{$\begin{array}{l}\text { Supplementing } \\
\text { breastfeeding }\end{array}$} & Before 6 months & 121 & $62 \%$ \\
\hline & After 6 months & 75 & $38 \%$ \\
\hline \multirow{2}{*}{$\begin{array}{l}\text { Frequency } \\
\text { breastfeeding }\end{array}$} & High $(>10)$ daily & 110 & $56 \%$ \\
\hline & Low $(<=10)$ daily & 86 & $44 \%$ \\
\hline \multirow{2}{*}{$\begin{array}{ll}\text { Intensiveness } & \text { of } \\
\text { breastfeeding } & \end{array}$} & Intensive ( $>5 \mathrm{~min})$. & 119 & $61 \%$ \\
\hline & Non inten. $(<=5 \mathrm{~min})$ & 77 & $39 \%$ \\
\hline
\end{tabular}

The main focus here is to use the survival analysis earlier discussed to estimate the probability that mothers who entered into the study will survive the transition from one state (non-amenorrhea state) to another state (amenorrhea state) and the risk involve in moving from one state to another state while breastfeeding, that is, to estimate time-to-event of the group of mothers involve in the research. 200 questionnaires were distributed to the respondents but only 196 were returned. Hence, the sample size used for this analysis is $\mathrm{N}=196$.

Table 1.2: Characteristics of Distribution obtain from MINITAB Characteristics Estimate standard Error

$$
\begin{array}{lcc}
\text { Mean } & 10.9388 & 0.7813 \\
\text { Standard dev. } & 10.988 & 0.7813 \\
\text { Median } & 7.5822 & 0.5416
\end{array}
$$

Table 1.3: Survival Probability Obtain from MINITAB Application of equation 6. Duration Probability Lower Upper (Months)

$\begin{array}{llll}3.0000 & 0.7601 & 0.7294 & 0.7879 \\ 7.0000 & 0.5273 & 0.4790 & 0.5733 \\ 11.0000 & 0.3658 & 0.3145 & 0.4172 \\ 15.0000 & 0.2538 & 0.2065 & 0.3036 \\ >15.0000 & 0.0000 & 0.0000 & 0.0000\end{array}$

Table 1.2 above gives the characteristics of the distribution which describes the distribution. The estimate of the mean, standard deviation and median of the distribution as while as their respective standard errors are given in the table. The survival function is assumed to approach zero as duration of PPA increases without bound, that is, $S(t) \rightarrow 0$ as $t \rightarrow \infty$. Table 1.3 above gives the various probabilities of surviving pass different durations without resuming menses that is, $P(T>t)=S(t)$ or more generally, the table gives the probability that the event of interest (resume menses) has not occurred by durations; $t=3,7,11,15$ and above 15 months respectively. Since amenorrhea will surely occur as $t$ get larger and larger, a point will be reach where $P(T=t)$ that is, probability of surviving pass that time will be equal to zero. Figure 1.2 gives the survival curve for the 196 subjects. 


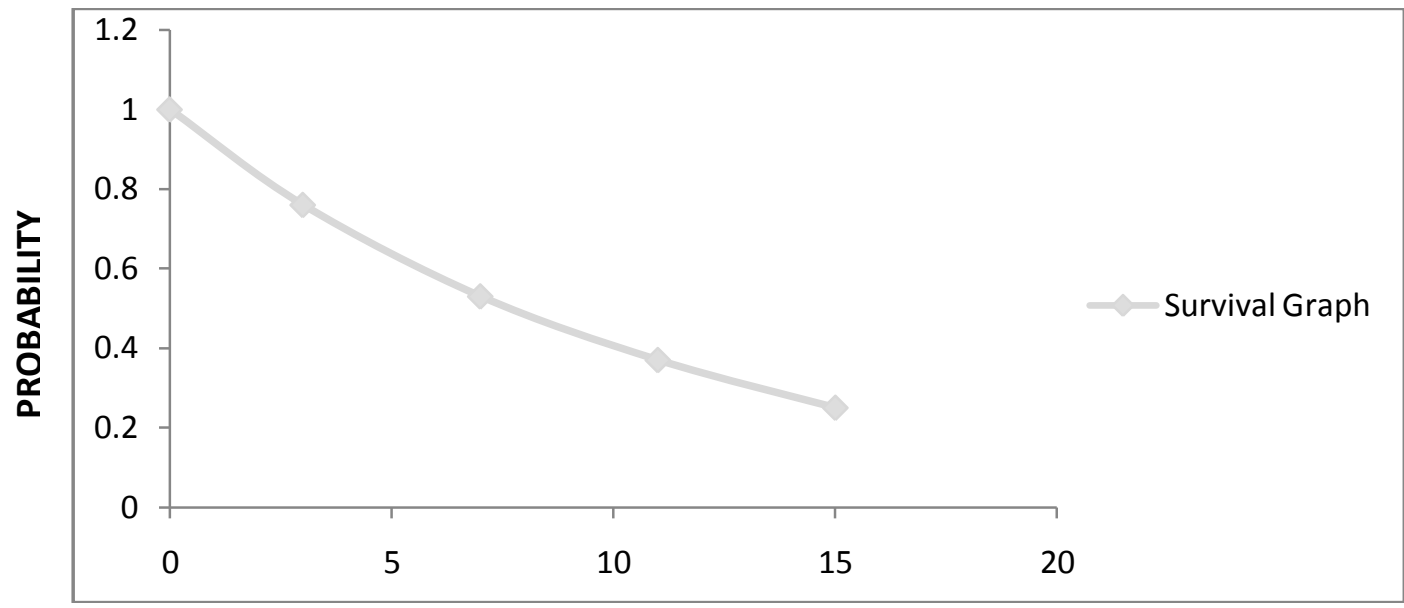

Figure 1.1: Survival graph of probability against duration in months from minitab result in Table 1.3.

Table 1.4: Empirical Hazard Probability from MINITAB Application of equation 2.

$\begin{array}{cc}\text { Duration(months) } & \text { Hazard Estimates } \\ 3.0000 & 0.006289 \\ 7.0000 & 0.008130 \\ 11.0000 & 0.009901 \\ 15.0000 & 0.02703 \\ >15.0000 & 1.000\end{array}$

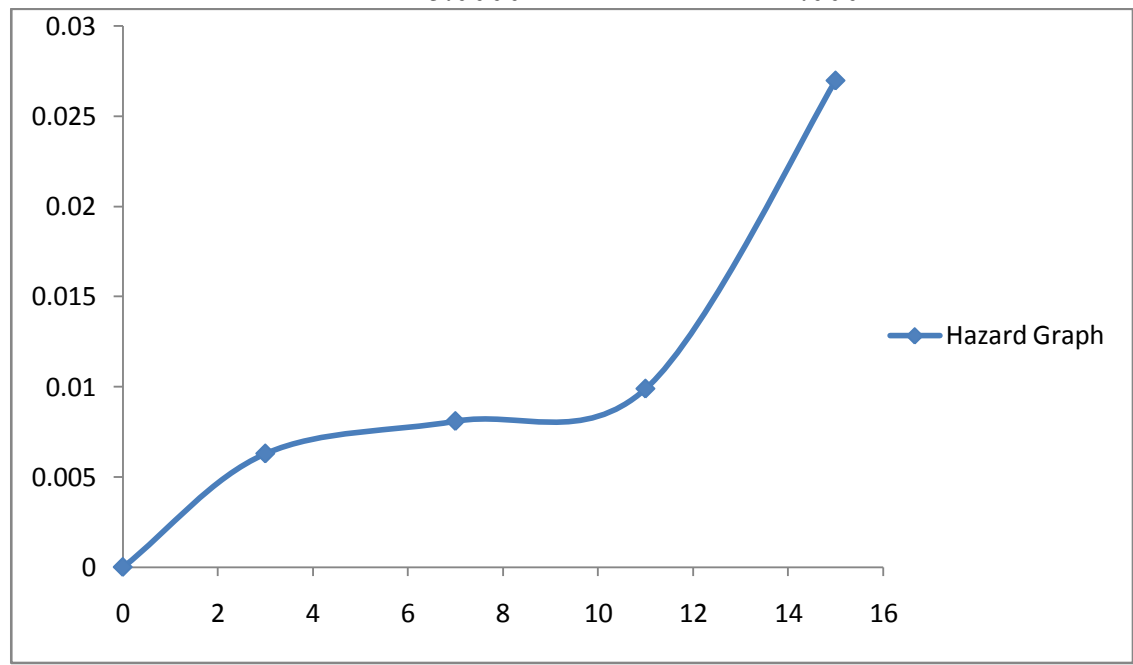

Figure 1.2 Hazard graph of probability against duration in months from minitab result of Table 1.4.

Table 1.4 gives the corresponding risk that if a subject survives up to a period $t$ without resuming menses; she will succumb to the event (resume menses) in the next period. The hazard function is seen to increase as the duration of breastfeeding increases, such that, at a certain period, survival becomes impossible and the event of interest (resume menses) must occur. Such as in the case of the duration of above 15 months where the survival is impossible at a certain period above the 15 months and so hazard is equal to one. Figure 1.1 gives the hazard curve for the 196 subjects.

Table 1.5: Kaplan-Meier Estimates

\begin{tabular}{ccccccc}
$\begin{array}{c}\text { Duration } \\
\text { (months) }\end{array}$ & $\begin{array}{r}\text { Number } \\
\text { at Risk }\end{array}$ & $\begin{array}{c}\text { Number } \\
\text { Failed }\end{array}$ & $\begin{array}{c}\text { Survival } \\
\text { Probability }\end{array}$ & $\begin{array}{c}\text { Standard } \\
\text { Error }\end{array}$ & \multicolumn{2}{c}{$95.0 \%$ Normal CI } \\
Lower & Upper \\
3.0000 & 196 & 38 & 0.8061 & 0.0282 & 0.7508 & 0.8615 \\
7.0000 & 158 & 36 & 0.6224 & 0.0346 & 0.5546 & 0.6903 \\
11.0000 & 122 & 22 & 0.5102 & 0.0357 & 0.4402 & 0.5802 \\
15.0000 & 100 & 64 & 0.1837 & 0.0277 & 0.1295 & 0.2379 \\
$>15.0000$ & 36 & 36 & 0.0000 & 0.0000 & 0.0000 & 0.000
\end{tabular}


The Kaplan- Meier estimates is a non- parametric method of estimating the fraction of subjects that survive pass a certain duration. Table 1.5 gives the MINITAB result for 196 subjects that entered into the study. For instance, the number of subjects that entered into the study from the beginning are 196, after three months, 38 subjects failed (had resume menses) hence the fraction of the subjects that survive pass the three months duration is $158 / 196$ and the corresponding probability that survive pass that duration is 0.8061 with standard error of 0.0282 . This is given for all the values of $t$. only 36 subjects survive pass 15 months without resuming menses, and at certain point $\mathrm{t}$ after 15 months, $\mathrm{s}(\mathrm{t})=0$ which means, survival becomes zero at some period $\mathrm{t}$. Figure 1.3 is the Kaplan-Meier graph for the 196 mothers that entered into the study.

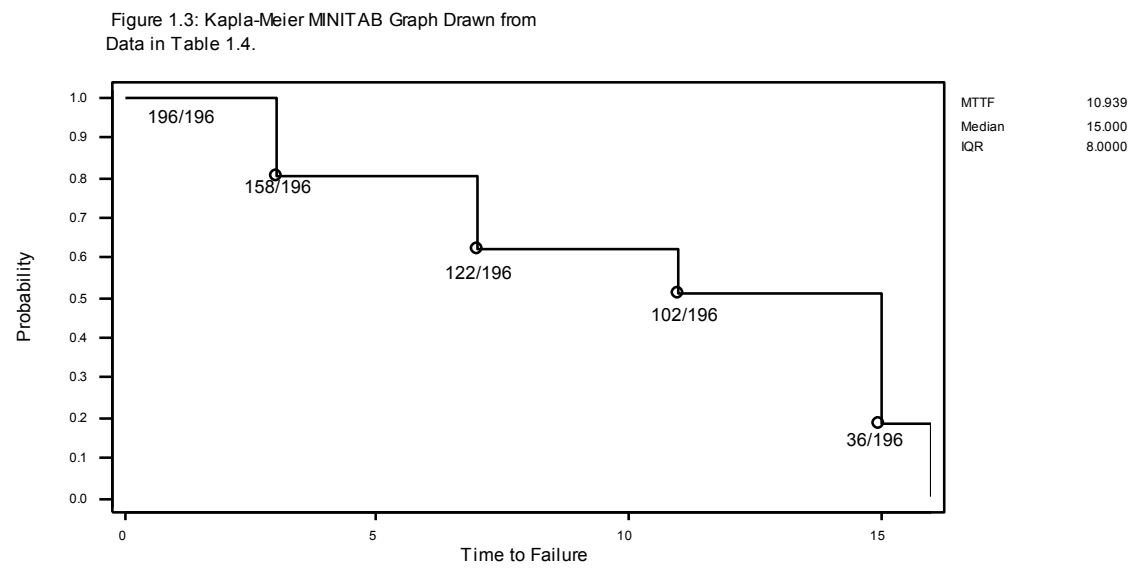

\section{Conclusion}

This research looked at how the survival analysis can be used to estimate the probability of breastfeeding women surviving to certain duration without resuming menses. Previous researches show that, breastfeeding and postpartum amenorrhea have significant effect on fertility of women since most women don't have their menses resumed until after weaning. A statistical package (Minitab) was applied on the Survival function and hazard function models (including the Kaplan-Meier method) and the result and graph obtained.

The research work revealed the following findings; that the age of mothers, occupation, and religion does not in any way affect the duration of postpartum amenorrhea. In estimating time-to-event, it was found that, as the duration $t$ of breastfeeding increases, the hazard function $h(t)$ of surviving without experiencing amenorrhea increases along while, survival function $s(t)$ of surviving without amenorrhea decreases as the duration increases. Some women had amenorrhea resumed long before breastfeeding is terminated regardless of their nutritional intake, frequency or intensity of breastfeeding. (For the purpose of this study, we referred to this group as the breastfeeding group). That most women in the breastfeeding group start taking family planning because they were exposed to pregnancy while still breastfeeding. That in the case of mothers in the weaning group (mothers who terminate breastfeeding before amenorrhea resume), breastfeeding is seen to be positively related to postpartum amenorrhea and as such, affect the fertility of the women and can be used as a natural means of birth control. It is a common practice among the rural Mada women to breastfeed their children for a long period normally for more than 12 months.

From the result obtained in this research, the association between breastfeeding and postpartum amenorrhea is not due to the direct effect of prolong lactation but as a result of some physiological mechanisms which involve the pituitary hormone prolactin (PRL) that varies from one woman to another. The age, religion and mothers occupation does not have any effect on the duration of postpartum amenorrhea. The time it takes to experience the first postpartum menses following the discontinuation of breastfeeding is approximately 1-2 months which is the same as when a woman had infant mortality or chooses not to breastfeed her child for any reasons. These confirm earlier findings from other similar studies. The estimation of time-to-event shows that, the chances of a woman surviving without amenorrhea decreases as duration of breastfeeding increase, while, the risk of surviving without amenorrhea increase as duration of breastfeeding increases.

\section{References}

[1] R.G. Potter, M.L. New, J.B. Wyon, and J.E Gordon, Application of Field Studies to Research on the Physiology of Human Reproduction: Lactation and its Effects on Birth Interval in Eleven Punjab Villages, India. Journal of chronic diseases 18: 1965, 1125-1140.

[2] R.E Jones, A Hazard Model Analysis of Breastfeeding Variables and Maternal Age on Return to Menses Postpartum in Rural Indonesian Women. Human Biology. 60: 1988, 853-871.

[3] United Nations (1991), Maternal and Child Care Development. Recommendation of Innocent Declaration on Breastfeeding Promotion (1990). United Nations: New York. 
[4] B. Figueiredo, C.C. Dias, R. Nunes-Coasta,, S. Brandao \& C. Canario, Breastfeeding and Postpartum Amenorrhea Depression: State of the Art Review. Journal of Pediatric. 13: 2013, S0021-7557.

[5] A.P. Makinde, Socio-Cultural Factors Affecting Fertility in Sub-Sahara Africa. Workshop on Prospects for Fertility Decline in High Fertility Countries. Population Division, Department of Economic and Social Affair. United Nation Secretariat. New York, 2001.

[6] A.G. Pinto, Longitudinal Analysis of the Risk of Resumption of Menses in Guatemalan. CDE Working Paper Centre for Demography and Ecology, University of Wisconsin- Madison. NO. 94: 1995, 23.

[7] J.P. Habicht, J. Davanzo, W.P. Butz, and L. Meyers, The Contraceptive Role of Breastfeeding. Population Study. 39: 1985, 213.

[8] K.B.S. Bonyata, Breastfeeding and Fertility. International Board Certificated Lactation Consultant (IBCLC), 2011.

[9] A.S.McNeilly, Lactation Control of Reproduction. Reproduction Fertility Development.13, 2001,583.

[10] M.M. Rahman, The Effect of Breastfeeding on Amenorrhea Attrition Rates in Rural Bangladesh. Journal of Biosocial Science 20: 2009, 32-35.

[11] M.M. Rahman, and S. Akter, Duration of Postpartum Amenorrhea Associated with Breastfeeding Pattern in Bangladesh. Journal of Sociology and Anthropology. 2: 2010, 011-018.

[12] A.G. Pinto, R.E. Jones, Breastfeeding and Postpartum Amenorrhea in Rural Guatemala: Mesoamerica Publication.3: $2005,2-3$.

[13] T.R. Aryal, Differential of Postpartum Amenorrhea. A Survival Analysis. Journal of Nepal Medical Association. 46: 2007 , 66-73.

[14] G. Santow, A Simulation Approach to the Study of Human Fertility. Nijhoff, Leiden, Holland 1978.

[15] A.G. Pinto, Breastfeeding and Postpartum Amenorrhea among in Bolivian Women: A Survival Analysis, CDE Working Paper,2005, Centre for Demography and Ecology, University of Wisconsin-Madison.

[16] G. Rodriguez, Generalized Linear Models. CDE Working Paper. No. 62, 2012. Office of Population Research, Princeton University.

[17] R. Singh, K. Mukhopadhyay, Survival analysis in Clinical Trials: Basics and Must Know Areas. Perspect Clinical Research. 2: 2011, $145-148$.

[18] K. Sainani, Application of Survival Analysis. Center for Health Research and Policy, Stanford University. Unpublished, 2013. 